\title{
Duration of the Operation Has No Effect on Early Visual Acuity After Small Incision Lenticule Extraction Surgery
}

\author{
Alper Agca, Yusuf Yildirim, Burcin Kepez Yildiz, Onur Olcucu, Dilek Yasa, Ali Demircan, Ahmet Demirok \\ Beyoglu Eye Training and Research Hospital, Istanbul, Turkey
}

\begin{abstract}
Objectives: The effect of the duration of small incision lenticule extraction (SMILE) operation on early postoperative visual acuity (VA) was evaluated.

Methods: Medical records of refractive lenticule extraction patients were retrospectively reviewed. Data on 38 eyes of 20 consecutive patients operated on by the same surgeon were included in the study. Primary outcome measures were corrected distance VA at postoperative first day and first week. Duration of the surgery was determined from video recordings of the operations.

Results: Mean patient age was $27.8 \pm 4.8$ years (range: $2 \mathrm{I}-40$ years). Mean preoperative spherical equivalent was $-4.38 \pm \mathrm{I} .4$. Postoperative distance corrected visual acuity was $0.7 \pm 0.1$ and $0.8 \pm 0.2$ at day $I$ and week $I$ visits, respectively. Mean duration of operation was $229.5 \pm 106$ seconds (range: $95-525$ seconds). No perioperative complications were seen. There was no statistically significant relationship between surgery time and postoperative VA at day I and week I visits.

Conclusion: Total time of operation does not have a statistically significant effect on early VA in SMILE patients.

Keywords: Femtosecond laser, small incision lenticule extraction, SMILE, visual acuity, visual rehabilitation.
\end{abstract}

\section{Introduction}

Refractive lenticule extraction (ReLEx) is a relatively new surgical procedure for correction of myopia and myopic astigmatism (I). It can be performed only with the VisuMax (Carl Zeiss Meditec AG, Jena, Germany) femtosecond laser platform. This platform provides ability to perform 2 types of ReLEx surgeries: femtosecond lenticule extraction (FLEx) and small incision lenticule extraction (SMILE). In SMILE procedure, an intrastromal lenticule is created with the femtosecond laser. Spatula inserted through side incision approximately $3 \mathrm{~mm}$ in length sweeps lamellar planes to dissect the tissue bridges connecting the lenticule to remaining stroma. The lenticule is then extracted from the side cut.

Results of SMILE are similar to FemtoLasik in terms of effectiveness and safety, and the concept behind both surger- ies is also quite similar (2). Intrastromal refractive lenticule is removed from the same depth of cornea in both. Visual recovery after SMILE is delayed, however, when compared with FemtoLasik (3-5). The reason for the delay is not well understood. The dissection of the lenticule is the most difficult and time-consuming part of the surgery. It could be argued that fewer surgical maneuvers, less trauma, or ease of removing the lenticule could create some difference in postoperative VA among SMILE patients. It can also be assumed that duration of the surgery is an indicator of the difficulty of lenticule dissection and thus, surgical trauma.

This study is an investigation of relationship between the duration of surgery performed by the same surgeon using the same dissection spatula (Chansue SMILE spatula G-33950, Geuder AG, Heidelberg, Germany) and early postoperative VA.

Address for correspondence: Alper Ağca, MD. Beyoglu Eye Training and Research Hospital, Bereketzade Cami Sok. 34421 Beyoglu, Istanbul, Turkey

Phone: +90 21225159 00, E-mail: agcaalper@yahoo.com

Submitted Date: October 19, 2016 Accepted Date: November 06, 2016 Available Online Date: November 15, 2016 ${ }^{\circ}$ Copyright 2016 by Beyoglu Eye Training and Research Hospital - Available online at www.beyoglueye.com 


\section{Methods}

Medical records of refractive lenticule extraction patients were retrospectively reviewed. Data regarding 38 eyes of 20 patients were included in the study. All patients were over 18 years of age with myopia or myopic astigmatism with spherical equivalent (SE) $<10 \mathrm{D}$, mesopic (4 lux) pupil size $\leq 6.5 \mathrm{~mm}$ and calculated residual stromal bed thickness $>300$ $\mu \mathrm{m}$, normal topographic pattern, regular retinoscopic reflex, corneal pachymetry $>500 \mu \mathrm{m}$ at the thinnest point, and stable refraction for at least 2 years.

Only patients who were operated on by the same surgeon (AD), had a complete video record of the surgery, and complete I day and I week visit data including measurements of distance corrected visual acuity (DCVA) were included. Patients who had a pre-existing eye disease or preoperative DCVA of less than 20/20, and patients who had an intraoperative or postoperative complication were excluded from the present research. In addition, if surgical spatula other than Chansue SMILE spatula G-33950 (Geuder AG, Heidelberg, Germany) was used, patient data was excluded.

Surgical videos of every eye operation included in the study were reviewed by the same author. Duration of the surgery was recorded and its correlation with postoperative VA was calculated using Pearson correlation analysis.

\section{Results}

Of the total, 9 participants were male and II were female. Preoperative patient characteristics are provided in Table I. Mean preoperative SE was $-4.38 \pm 1.4$ and mean postoperative SE was $-0.25 \pm 0.43$ (paired samples t-test; $p<0.05$ ). The same optical zone and energy parameters were used for all eyes (Table 2). Postoperative DCVA was $0.7 \pm 0.1$ and $0.8 \pm 0.2$ at day I and week I visits, respectively (paired samples t-test; $p<0.05$ ). Mean duration of operation was $229.5 \pm 106$ seconds (range: 95-525 seconds). There was no statistically significant relationship between length of the operation and postoperative VA at day I and week I visits (Table 2; Pearson's correlation analysis; $\mathrm{p}>0.05$ ).

\section{Discussion}

SMILE is a relatively new alternative to LASIK for the correction of myopia. The concept behind SMILE surgery is very similar to femtosecond laser-assisted LASIK because intrastromal lenticule is removed from the eye in both procedures. However, VA is delayed in SMILE patients compared to those who undergo LASIK procedure (3-5). In addition, some patients have an unpredictable loss of more than 2 DCVA lines in the first few weeks after SMILE. Shah et al. (6) reported that only $58 \%$ of patients had I-week postoperative uncorrected distance visual acuity (UDVA) better than
Table I. Preoperative characteristics

Mean \pm SD

Age (years)

$27.8 \pm 4.8$

Keratometry

$44.7 \pm 2.4$

Pachymetry

$532 \pm 29$

Refractive error

$-4.38 \pm 1.4$

SD: Standard deviation.

Table 2. SMILE parameters

Optical zone (mm)

6.5

Side cut $(\mathrm{mm})$

3

Side cut location (quadrant)

Spot energy (nJ)

Superior

Spot separation $(\mu)$

140

3.5

SMILE: small incision lenticule extraction.

Table 3. Correlation between duration of the surgery and visual acuity CDVA (Day I) CDVA (Week I)

Duration $r=0.34(p>0.05)$ $r=0.2(p>0.05)$

CDVA: corrected distance visual acuity. r: Pearson's correlation coefficient.

or equal to preoperative CDVA, though this figure improved to $86 \%$ at 6 months. They concluded that visual recovery is delayed in lenticule extraction when compared with best results of modern refractive surgery. In the present study, mean postoperative CDVA was better at first week compared to day I $(p>0.05)$. However, CDVA was only $0.8 \pm 0.2$ at week I though all patients had CDVA of at least I.0 preoperatively. Our results are consistent with other studies in the literature in finding that early VA was not as good in SMILE patients when compared to LASIK patients.

While some patients do not experience a loss of CDVA after undergoing SMILE, some patients lose more than 2 or 3 lines of VA. This phenomenon is more pronounced in the earliest days and vision gradually recovers in a few weeks or months. However, it is not possible to predict which eyes will have better early postoperative VA. It could be argued that although the lenticule extraction is the same, fewer surgical maneuvers, less traumatic surgery, or ease of removing the lenticule could create some difference in postoperative VA among SMILE patients. To test this hypothesis, it was assumed that the difficulty the surgeon encounters and an increase in the number of surgical maneuvers would have an effect on the duration of the surgeries performed by that single surgeon if all remaining parameters such as the surgical instrument used remained the same. Thus, we evaluated the 
surgeries of only I experienced surgeon and recorded the exact duration of the procedures based on video records. After watching the videos, we believe that it is not wrong to assume that the duration of surgery reflects the number of surgical manipulations and the ease of dissection (for that single surgeon) in this patient group. However, although the duration of the surgeries (range: 95-525 seconds) and postoperative VA were distributed in a wide range, there was no statistically significant correlation between operation time and early VA at day I or week I.

Although the same lamellar cuts are performed with the same femtosecond laser platform and similar lenticule is removed in both SMILE and FLEx surgery, the FLEx procedure requires fewer surgical maneuvers and therefore creates less trauma to the underlying stroma. Thus, comparing VA of SMILE and FLEx patients is another way of evaluating the effects of surgical maneuvers or surgery time on VA. Agca et al. previously compared SMILE and FLEx surgeries in a small but matched group of eyes (7). Their results indicated that FLEx surgery does not offer better VA compared to SMILE, and this supports the findings of the present study.

This study has important limitations. One limitation is that there were too few patients to make strong assumptions. In addition, duration of the surgery may not reflect the number of surgical manipulations or stromal trauma that occurs during surgery. Thus, stromal trauma during dissection may be a factor in loss of CDVA in early postoperative period and this study may have lacked sufficient power or the correct design to reveal such a relationship. However, after observing that some fellow eyes with similar preoperative characteristics, similar surgical maneuvers performed by the same surgeon, similar duration of surgery, and no intraoperative complications had very different surgical outcomes in the early postoperative period ( I eye may lose 2-3 lines while fellow eye does not lose any lines), we believe that there are other factors leading to loss of CDVA in the early postoperative period. It can be argued that I such factor may be related to the quality and regularity of femtosecond laser cuts on anterior or posterior surfaces of the lenticule.

In conclusion, we did not find a significant correlation between duration of the surgery and VA achieved in the early postoperative period. The results of this study indicate that surgery time is not a major risk factor for loss of CDVA in uncomplicated SMILE surgery.

\section{Disclosures}

Financial Disclosure: No author has a financial or proprietary interest in any material or method mentioned. These data have not been previously published.

Peer-review: Externally peer-reviewed.

Conflict of Interest: None declared.

\section{References}

I. Sekundo W, Kunert K, Russmann C, Gille A, Bissmann W, Stobrawa $G$, et al. First efficacy and safety study of femtosecond lenticule extraction for the correction of myopia: six-month results. J Cataract Refract Surg 2008;34:1513-20. Crossret

2. Vestergaard A, Ivarsen AR, Asp S, Hjortdal JØ. Small-incision lenticule extraction for moderate to high myopia: Predictability, safety, and patient satisfaction. J Cataract Refract Surg 2012;38:2003-10. Erossre

3. Vestergaard A, Ivarsen A, Asp S, Hjortdal JØ. Femtosecond (FS) laser vision correction procedure for moderate to high myopia: a prospective study of $\operatorname{ReLEx}(\circledR)$ flex and comparison with a retrospective study of FS-laser in situ keratomileusis. Acta Ophthalmol 2013;91:355-62. Erossre

4. Kamiya K, Shimizu K, Igarashi A, Kobashi H, Komatsu M. Comparison of visual acuity, higher-order aberrations and corneal asphericity after refractive lenticule extraction and wavefront-guided laser-assisted in situ keratomileusis for myopia. $\mathrm{Br}$ J Ophthalmol 2013;97:968-75. Crossre

5. Gertnere J, Solomatin I, Sekundo W. Refractive lenticule extraction (ReLEx flex) and wavefront-optimized Femto-LASIK: comparison of contrast sensitivity and high-order aberrations at I year. Graefes Arch Clin Exp Ophthalmol 2013;25I:143742. Crossre

6. Shah R, Shah S, Sengupta S. Results of small incision lenticule extraction: All-in-one femtosecond laser refractive surgery. J Cataract Refract Surg 201 1;37:127-37. Crossre

7. Ağca A, Demirok A, Cankaya Ki, Yaşa D, Demircan A, Yildirim $Y$, et al. Comparison of visual acuity and higher-order aberrations after femtosecond lenticule extraction and small-incision lenticule extraction. Cont Lens Anterior Eye 2014;37:292-6. 